International Journal of Performability Engineering, Vol. 13, No. 1, January 2017, pp. 95-106

(C) Totem Publisher, Inc., 4625 Stargazer Dr., Plano,

Printed in U.S.A.

\title{
A Two Step Method for Tower Structure Damage Location
}

\author{
YU ZHEFU $^{* 1}$ and HUO LINSHENG ${ }^{2}$ \\ ${ }^{1}$ Dalian Maritime University, Transportation Equipment and Marine Engineering \\ College, Linghai Road No.1, Liaoning, Dalian, Co. 116026, PRC \\ ${ }^{2}$ Dalian University of Technology \\ Linggong Road No.2, Integrated Building 4, 219-B, Liaoning, Dalian, Co.116024, PRC
}

(Received on October18, 2016 revised on December 13, 2016)

\begin{abstract}
For a tower structure containing many bars, the possible number of damage patterns in a tower is numerous. To solve this problem, a new two-step method was proposed based on a cross-correlation function and second generation wavelets. First step, by comparing the peaks of cross-correlation function, damage basic units can be found. Second step, we select a suitable second generation wavelet for distinguishing damage patterns with vector angle similarity measure. SVM are used to pinpoint damage bar last. Test result indicates that this method has good locating accuracy.
\end{abstract}

Keywords: iron tower; cross-correlation function; damage location; second generation wavelets; vector angle similarity measure.

\section{Introduction}

In the past two decades, structure-damage-locating methods based on vibration analysis have become prevalent [1, 2]. In recent years, damage-locating methods that integrate time-series processing and artificial intelligence have progressively attracted the attention of researchers [3]. The basic idea of such methods is described here. The vibration responses obtained using the finite element method and corresponding damage patterns constitute a set of training samples. Usually, the vibration responses require some kind of analysis. One uses these samples to train a learning machine. Subsequently, one use the learning machine to distinguish damage patterns according to the vibration responses obtained in a field. Currently, these methods are only used in some simple structures that have only a small number of bars $[4,5]$.

Tower structures are subject to all types of damages. Locating damages quickly is crucial for its maintenance. This study proposes a two-step damage location method for the tower structures which have a large number of bars. First step, we use the crosscorrelation functions to identify coarse damage locations. Second step, we use the second generation wavelets to pinpoint damage bars.

\section{Coarsen damage location of tower structure}

\subsection{The conception of cross-correlation function}

Cross-correlation function can reflect the relationship of the two random vibration signals, and effectively avoid the noise pollution in the test signal of vibration response [6]. If a structure exposes to the environmental pulsation, the responses of two adjacent 
testing points can be regarded as two real stationary ergodicity random processes who are expressed as two discrete time series, $x_{1}(i)$ and $x_{2}(i)$. The cross-correlation function between them is shown in Eq.1.

$$
\varphi_{x_{1} x_{2}}(k)=\frac{1}{N} \sum_{i=1}^{N-k} x_{1}(i) x_{2}(i+k), \quad(k=0,1, \cdots N)
$$

Where $N$ denotes the number of signal sampling points.

The maximum of the cross-correlation value, as shown in Eq.2, is referred to as the peak in this paper. The peaks of the cross-correlation function are closely related to structure damages. Based on the peak mutations, the damage locations can be determined [7].

$$
r_{l, l+1}=\left|\varphi_{x_{l} x_{l+1}}(\tau)\right|_{\max }=\left|\varphi_{x_{l} x_{l+1}}(k)\right|_{\max }
$$

\subsection{Tower structure}

In this study, a tower structure was used to verify the damage location method, as shown in Fig. 1. The software SAP2000 was used for finite element analysis. Damage bars were simulated by decreasing the elastic modulus by $30 \%$ or $20 \%$.

Because wind loads have a great impact on the tower which belongs to high-rising structures, we selected environmental pulsation as the exciting vibration signal. In finite element analysis, the exciting vibration signal is a white noise generated by MATLAB based on environment pulsation parameters. Assuming acceleration amplitude delivered to the tower is $2 \times 10^{-3} \mathrm{~m} / \mathrm{s}^{2}$, the sampling interval is $0.01 \mathrm{~s}$, the sampling time is $10 \mathrm{~s}$. Exciting vibration signal acts on the tower through four fixed brackets.

Based on the characteristics of the tower structure, the acceleration sensors were fixed in the longitudinal direction of each chord hinge junction. That means that the acceleration sensors located in 8 corner points of every basic unit shown in Fig.2. The sensors were numbered sequentially from the bottom to the upper. The measured acceleration response is in the same direction.

\subsection{Amplitude vector}

The peak of cross-correlation function $r_{2,2-3}$ was calculated by using two adjacent acceleration response signals in the same corner, shown in Fig.2. This study proposed the concept that is the peak vectors of cross-correlation functions. For the tower, the peak vectors from four corners are shown in Eq.3.

$$
\begin{aligned}
& R_{1}=\left|r_{1,1-2}, r_{1,2-3} \cdots r_{1,14-15}\right| \\
& R_{2}=\left|r_{2,1-2}, r_{2,2-3} \cdots r_{2,14-15}\right| \\
& R_{3}=\left|r_{3,1-2}, r_{3,2-3} \cdots r_{3,14-15}\right| \\
& R_{4}=\left|r_{4,1-2}, r_{4,2-3} \cdots r_{414-15}\right|
\end{aligned}
$$




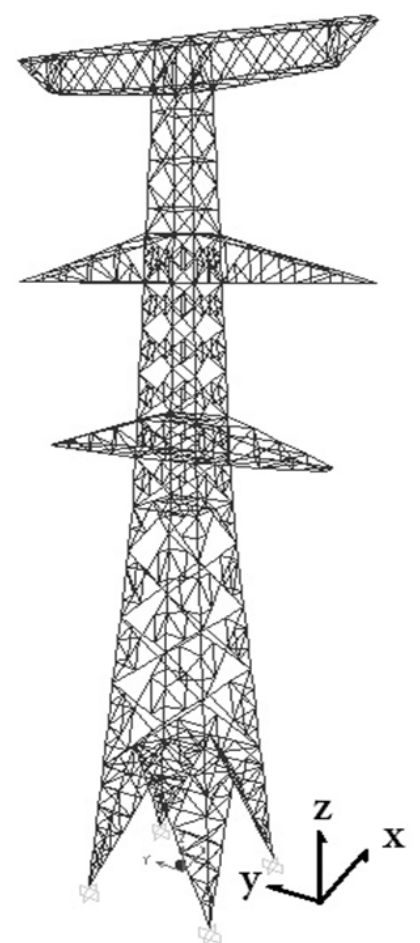

Fig. 1: Complete tower structure

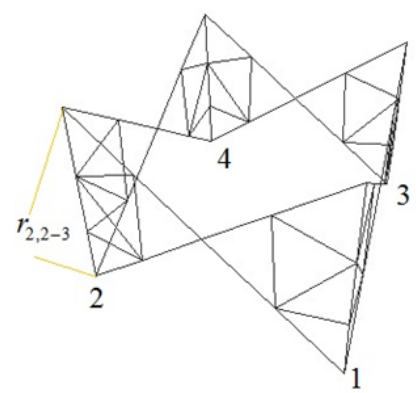

Fig. 2: Tower basic unit

Subscript 2 in $r_{2,2-3}$ denotes the fact that the cross-correlation function peak is situated in second corner. Subscript 2-3 in $r_{2,2-3}$ denotes the fact that the crosscorrelation function peak is obtained from the sensor in second layer and the sensor in third layer. $R_{2}$ denotes a peak vector for second corner.

We could use the mutations of peaks to find the locations damages occurred. If the damage position is in the second basic unit, the mutations of peaks are shown In Fig. 3-6. 
The solid lines are the peak vectors of the intact tower. The dash lines are the peak vectors of the damage tower. Comparing the peak vectors of damage structure with the peak vectors of intact structure, the position from where the peaks are different could be found.

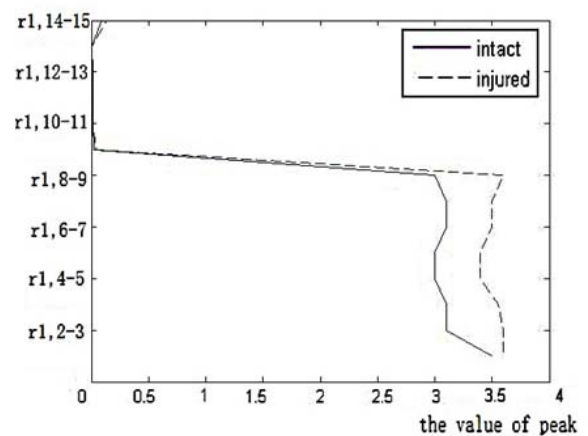

Fig.3: Peak vector of no.1 corner

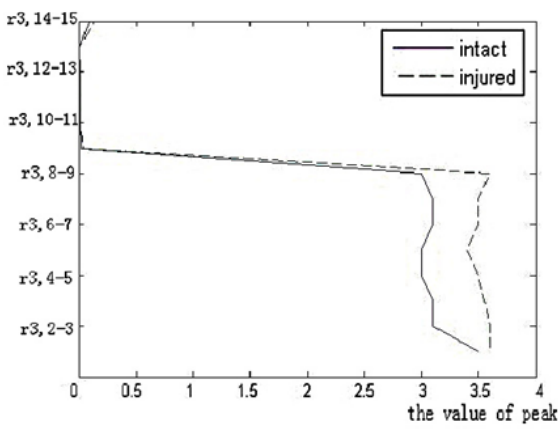

Fig.5: Peak vector of no.3 corner

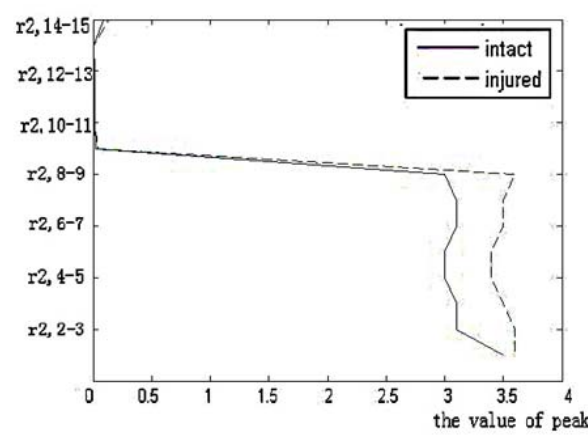

Fig.4: Peak vector of no.2 corner

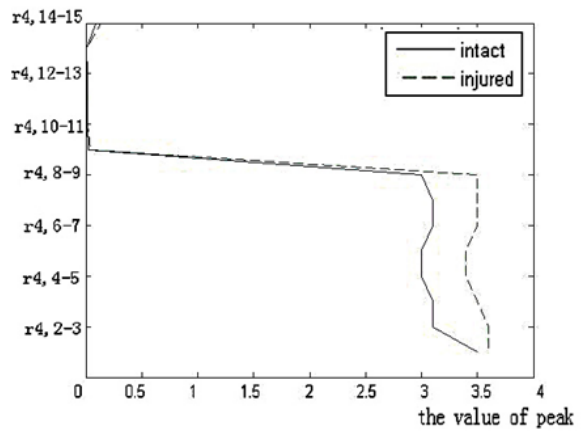

Fig.6: Peak vector of no.4 corner

The cross-correlation function also could be calculated by using two adjacent acceleration response signals in the same layer. In this case, the peak vectors are shown in Eq.4.

$$
\begin{aligned}
& R_{1-2}=\left|r_{1-2,1}, r_{1-2,2} \cdots r_{1-2,15}\right| \\
& R_{1-3}=\left|r_{1-3,1}, r_{1-3,2} \cdots r_{1-3,15}\right| \\
& R_{2-4}=\left|r_{2-4,1}, r_{2-4,2} \cdots r_{2-4,15}\right| \\
& R_{3-4}=\left|r_{3-4,1}, r_{3-4,2} \cdots r_{3-4,15}\right|
\end{aligned}
$$

Subscript 1-2 in $r_{1-2,1}$ denotes the fact that the cross-correlation function peak is obtained from the sensor in first corner and the sensor in second corner. Subscript 1 in $r_{1-2,1}$ denotes the fact that the cross-correlation function peak is situated in first layer. $R_{2}$ denotes a peak vector for second corner.

From Fig. 7-10, comparing the peak vectors of the damage structure with the peak vectors of the intact structure, the damage position also could be found in the second basic unit. One thing to note, this basic unit is different with the basic unit shown in Fig. 2. This 
basic unit is shown in Fig. 11. Integrating the distinguishing results of two kinds of vector, we can more precisely distinguish the damage location in the half basic unit shown as Fig.12.

The peak vectors obtained from the four corners are similar for one damage pattern. Judging the damaged area could be based on only one vector, so testing points can be arranged just along one or two corners. Damage positioning accuracy is a rate of $100 \%$, but the method cannot distinguish which bar occurred damage.

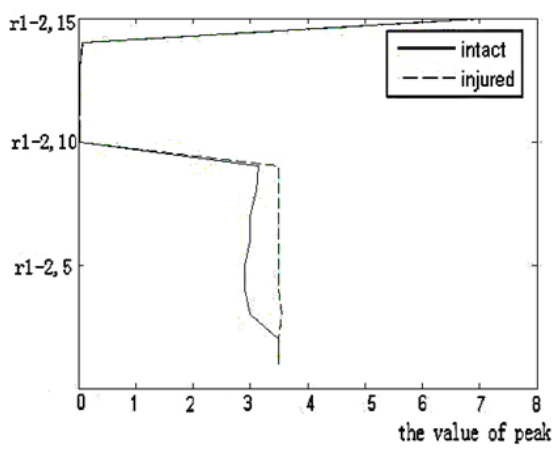

Fig.7: Peak vector from no.1 corner no. 2 corner

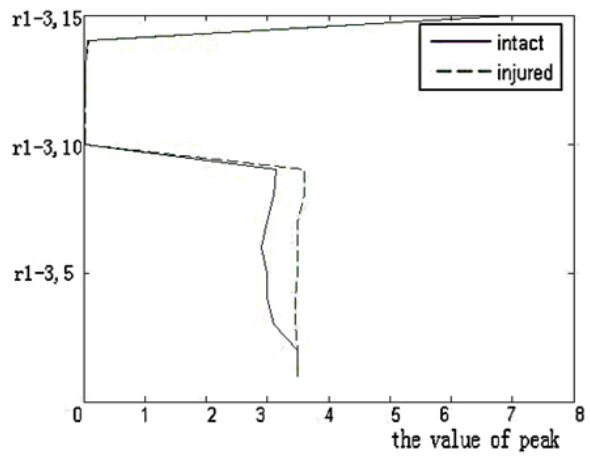

Fig.9: Peak vector from no.1 corner and no.3 corner

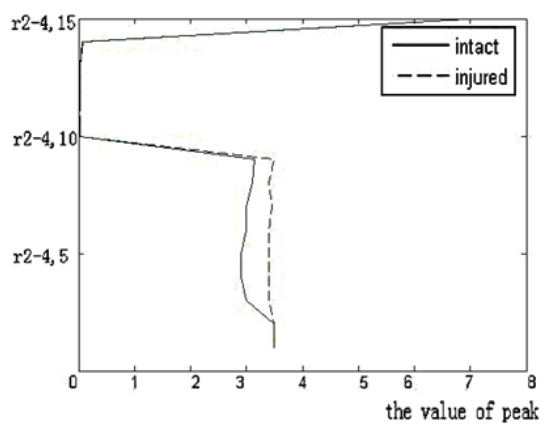

Fig.8: Peak vector from no.2 corner and no.4 corner

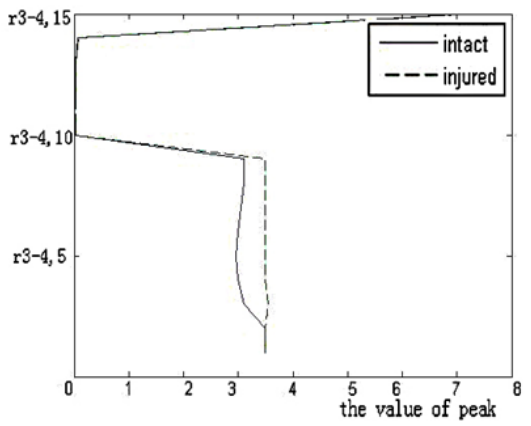

Fig.10: Peak vector from no.3 corner and no.4 corner 


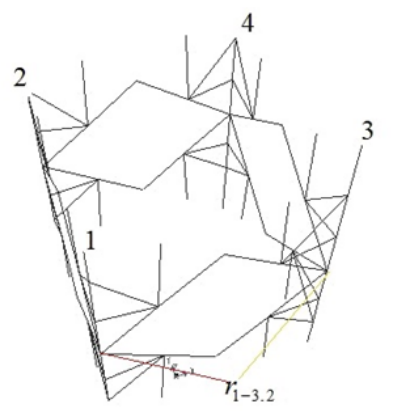

Fig. 11: Tower basic unit

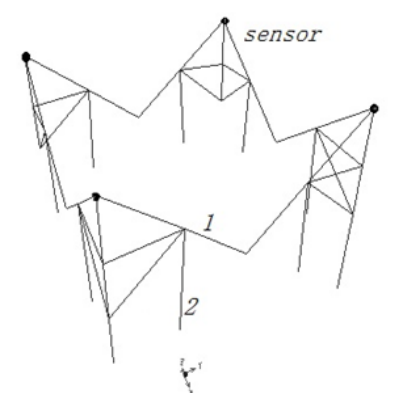

Fig. 12: Half of basic unit

\section{$2.4 \quad$ Processing peak vector}

For the upper truss of the tower, the basic unit has changed as Fig.13. It is different with the lower truss. The damage locations are not easy to find by using the crosscorrelation function peak mutation. That can be seen in Fig. 14. After the vector from a damage grid was subtracted by the vector from an intact grid, a new vector is obtained as shown in Fig.15. We can more easily identify the damage location in No. 12 basic unit.

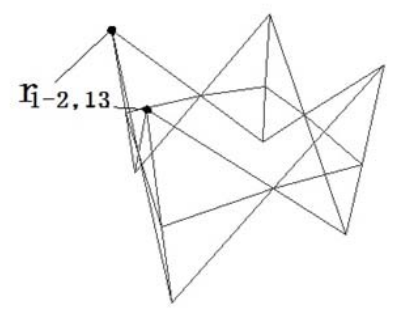

Fig. 13: Tower upper basic unit

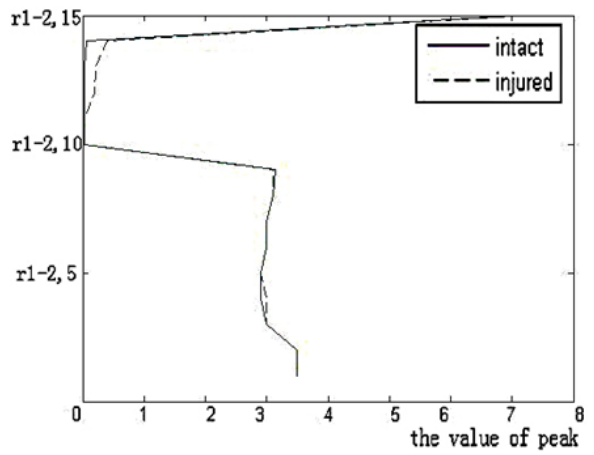

Fig. 14: Peak vector of an upper damage tower

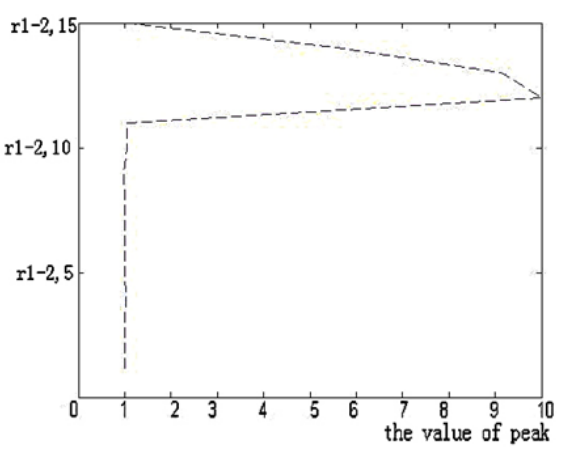

Fig. 15: Processed peak vector of an upper damage tower 
For one damage pattern of the tower, the peak vectors obtained from different vibrations under the same spectrum are highly similar [7, 8]. After normalizing, they are almost identical. Different damage patterns bring different peak vector. The environment pulsation is relatively stable. Its spectrum is almost unchanged under normal conditions. For same damage patterns, the peak vectors obtained at different time are highly similar. When the number of acceleration sensor is limited, we can get the whole peak vector also by repeatedly arranging the sensors in different zones.

\section{Pinpointing damage bars}

We can easily identify the damage unit by peak vector. To identify the damage bars in the unit, we introduced the second generation wavelets.

\subsection{Second generation wavelets. [9]}

During the last decade, many constructions of wavelets on the real line became available, see for example [10, 11]. The filter sequences for scaling functions and wavelets are typically derived through the Fourier transform and the consideration of certain trigonometric polynomials and their properties. From a user's point of view though, the constructions are not always suitable for straightforward implementation or specialization to particular cases, such as boundaries. In damage identification, the response signal is a finite length.

Literatures $[12,13]$ show that we can construct versatile families of scaling functions and wavelets under the techniques of interpolating subdivision [14] and lifting [15, 16]. To ingrate these techniques results in a straightforward and easy to implement toolkit. The wavelet constructed by this way belongs to general framework of "second generation wavelets.”

We begin with the construction of scaling functions by interpolating subdivision. All the algorithms can be derived via simple arguments involving nothing more than polynomial interpolation. The interpolating subdivision scheme can formally be defined as follows.

In signal $x_{i}$, the position of $N$ consecutive points is expressed as $t_{k+1}, t_{k+2}, \cdots t_{k+N}$, ( $N=2 D, D$ is a positive integer) and the signal value is expressed as $x_{i, k+1}, x_{i, k+2}, \cdots x_{i, k+N}$. A polynomial of degree $N-1$ exists and is expressed as $L(t)$, the polynomial satisfies the condition, $L\left(t_{k+l}\right)=x_{i, k+l}, l \in\{1,2, \cdots N\}$. Calculating signal value, $X_{i, k+D+1 / 2}$, in the middle point, $t_{k+D+1 / 2}$, of $N$ consecutive points with the polynomial $L(t)$, we find that the value, $x_{i, k+D+1 / 2}$, is equal to the weighted sum of $x_{i, k+1}, X_{i, k+2}, \cdots x_{i, k+N}$. Corresponding to different value of $N$, the weight coefficients for the series $x_{i, k+1}, x_{i, k+2}, \cdots x_{i, k+N}$ are shown in the table below [17]. 
Table1: The weight coefficients for the series $X_{i, k+1}, X_{i, k+2}, \cdots X_{i, k+N}$

\begin{tabular}{|c|c|c|c|c|c|c|c|c|}
\hline$N$ & $P_{1}$ & $P_{2}$ & $P_{3}$ & $P_{4}$ & $P_{5}$ & $P_{6}$ & $P_{7}$ & $P_{8}$ \\
\hline 2 & 0.5 & 0.5 & & & & & & \\
\hline 4 & -0.0625 & 0.5625 & 0.5625 & -0.0625 & & & & \\
\hline 6 & 0.0117 & -0.0977 & 0.5859 & 0.5859 & -0.0977 & 0.0117 & & \\
\hline 8 & -0.0024 & 0.0239 & -0.1196 & 0.5981 & 0.5981 & -0.1196 & 0.0239 & -0.0024 \\
\hline
\end{tabular}

Lifting scheme includes three steps: split, predict and update.

The first step is to split the original signal $x_{j}$, in accordance with the sampling sequence. One breaks down $x_{j}$ into its even part, $x_{j, 2 l}$ and odd part, $x_{j, 2 l+1}$, specific representation is shown as Eq.5.

$$
x_{j, 2 l}=\operatorname{even}\left(x_{j}\right) \quad x_{j, 2 l+1}=\operatorname{odd}\left(x_{j}\right)
$$

The second step is prediction. One uses even part $X_{j, 2 l}$ to predict odd part $X_{j, 2 l+1}$. Predicting operator is $P\left(x_{j, 2 l}\right)$. Prediction error $d_{j-1}^{\prime}$ is obtained as Eq.6.

$$
d_{j-1}^{\prime}=x_{j, 2 l+1}-P\left(x_{j, 2 l}\right)
$$

The third step is update. After converting prediction error $d_{j-1}^{\prime}$ by updating operator, $U\left(d_{j-1}^{\prime}\right)$, one updates the even part to obtain new low-frequency component, specifically calculated as Eq.7.

$$
x_{j-1}^{\prime}=x_{j, 2 l}+U\left(d_{j-1}^{\prime}\right)
$$

In the lifting scheme, interpolation subdivision principles were used to design predicting operator and updating operator. For some second generation wavelet, the prediction coefficients are same as the weight coefficients shown in Tab.1. Usually, Update coefficients are half of predictor coefficients. After updating operator and predicting operator are determined, scaling function and wavelet function are generated by iteration. In this study, we call the wavelets in Tab.1 as "SGW2, SGW4, SGW6, SGW8" from top to bottom.

\subsection{Vector angle similarity measure}

This paper introduces vector angle similarity measure to choose a suitable wavelet. For example, the acceleration responses in damaged structure were decomposed into 8 frequency components from low to high. After calculating the energy of every band, an 8dimensional feature vector is obtained. Then being normalized, it is shown in Eq.8.

$$
A_{i}=\left[E_{i, 0}, E_{i, 1}, E_{i, 2}, \cdots E_{i, 7}\right]
$$

An angle measure whose expression is defined as Eq.9.can be used to measure the similarity of two vectors. 


$$
S\left(A_{i}, A_{j}\right)=\frac{\left\langle A_{i}, A_{j}\right\rangle}{\sqrt{\left\langle A_{i}, A_{i}\right\rangle\left\langle A_{j}, A_{j}\right\rangle}}
$$

$\left\langle A_{i}, A_{j}\right\rangle$ denotes the inner product of two vectors. $S\left(A_{i}, A_{j}\right)$ denotes a cosine of the angle between the two vectors. The larger the value of $S\left(A_{i}, A_{j}\right)$ is, the smaller the angle between two vectors is. Namely, the two vectors are more similar. For example, in damage identification, when No. $i$ bar is damaged, the feature vector is $A_{i}$; when No. $j$ bar is damaged, the feature vector is $A_{j}$. The suitable wavelet makes $S\left(A_{i}, A_{j}\right)$ as large as possible, as shown in Fig.16. This wavelet makes the two vectors more discernible for classifier.

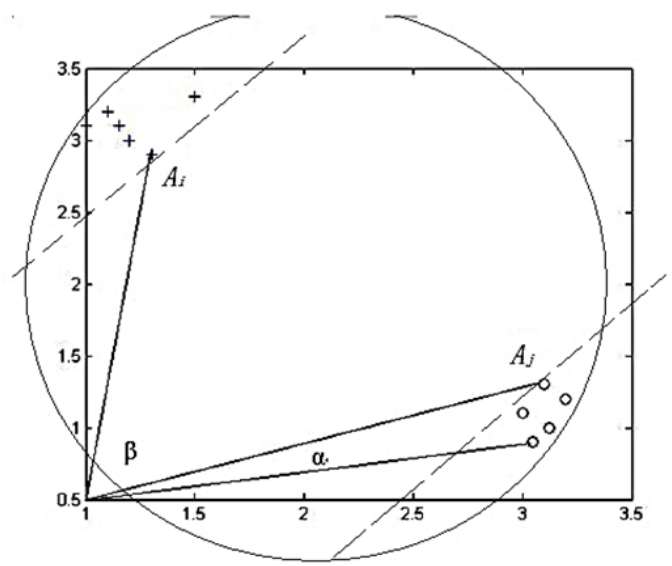

Fig. 16: Abridged general view of vector angle similarity measure

More specifically, for a vector set, $\left\{A_{i}^{\prime}, A_{i}^{\prime \prime}, A_{j}^{\prime}, A_{j}^{\prime \prime}\right\}$, the vectors with different subscripts means that they are derived from different damage patterns; the vectors with different superscripts means that they are derived from different damage degrees of same damage location. Good wavelet makes the value of Eq.10 maximum. Eq.10 can be used as a criterion when one selects wavelet function with program.

$$
F\left(A_{i}^{\prime}, A_{i}^{\prime \prime}, A_{j}^{\prime}, A_{j}^{\prime \prime}\right)=\frac{S\left(A_{i}^{\prime}, A_{j}^{\prime}\right) S\left(A_{i}^{\prime \prime}, A_{j}^{\prime \prime}\right) S\left(A_{i}^{\prime}, A_{j}^{\prime \prime}\right) S\left(A_{i}^{\prime \prime}, A_{j}^{\prime \prime}\right)}{S\left(A_{i}^{\prime}, A_{i}^{\prime \prime}\right) S\left(A_{j}^{\prime}, A_{j}^{\prime \prime}\right)}
$$

\subsection{SVM}

In this study, SVM was introduced to distinguish different damage patterns. SVM proposed by Vapnik is a machine learning algorithm based on statistical learning theory. It minimizes actual risk through seeking minimal structural risk. It can get a good learning result in the case of small sample size. Its solution is globally optimal. Classical SVM algorithm is a two-class classifier only, but general problems need multi-class classifiers. 
It was solved by combining multiple two-class classifiers. In this paper, the combination method of one to one is used to realize the multi-class classification.

\subsection{Distinguish damage bar}

Now, we should distinguish the damage bar in the half of basic unit shown in Fig.12. Considering the node situation, each half of basic unit has 36 damage patterns.

The acceleration responses from sensor shown in Fig. 12 were decomposed into 8dimensional feature vectors with second generation wavelet. After introducing vector angle similarity measure to choose a suitable wavelet, then, we select SGW8 as wavelet in this study. The feature vector of the intact base unit is shown in Fig. 17. For intact base unit, a long feature vector constituted with the feature vectors of four sensors in Fig. 12 are shown from Fig. 18. For damage base unit, the long feature vectors are shown from Fig. 19- 20.

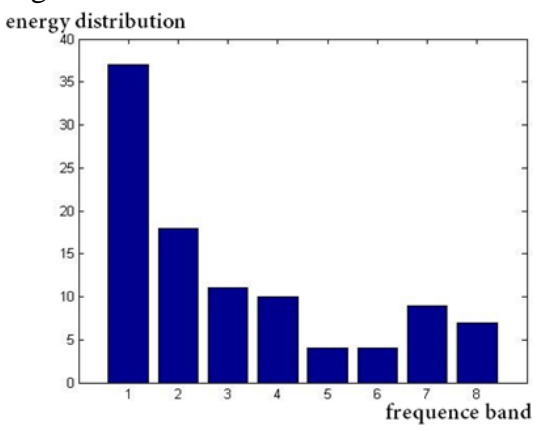

Fig. 17: Feature vector of the intact base unit

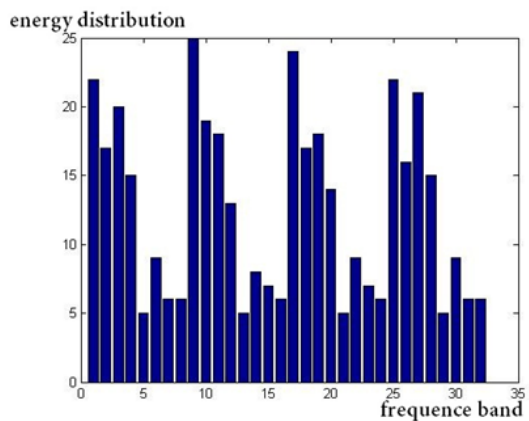

Fig.19: Long feature vector of the base unit with No.1damaged bar, shown in Fig. 12

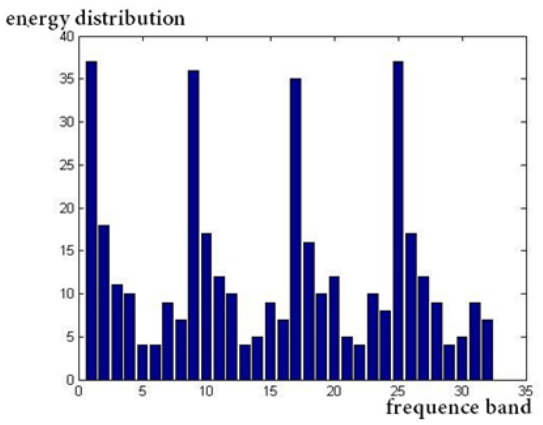

Fig.18: Long feature vector of the intact base unit

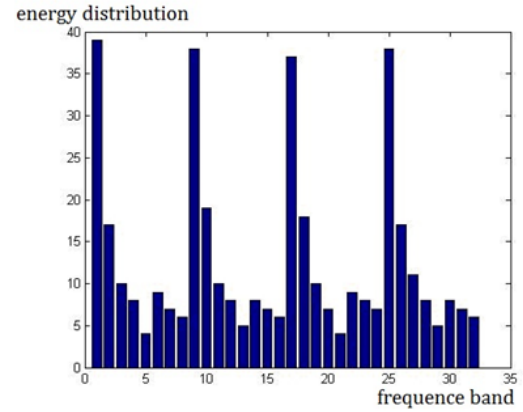

Fig.20: Long feature vector of the base unit with No.2damaged bar, shown in Fig. 12

A sample for SVM was composed of a long feature vector and a corresponding damage pattern. We collected 200 acceleration response signals as sample, which include every damage patterns. Four samples in each damage pattern are selected as the training set. In the SVM, the kernel function is RBF. The optimal $C$ and gamma were obtained by 4-fold cross-validation. The remaining 56 samples were used as test set. 
The testing accuracy is $85.7 \%$. When we use SGW2, SGW4 and SGW6, the testing accuracy is $64.3 \%, 71.4 \%$ and $75 \%$ respectively. It can be seen that the wavelet selected with vector angle similarity measure has the highest localization damage accuracy. This means that vector angle similarity measure is a good criterion for selecting wavelet. It can be used in similar wavelet selection problem.

There exists a method in the literature [18] that only selected main vertical bars in tower as the identification unit. It cannot identify all the damage bars. There exists a method in the literature [19] that needs an enormous number of training samples for a grid. There exists a method in the literature [20] that can deal with multiple-bar damage in a grid. The method use first generation wavelets, the accuracy of damage location is not high. When choosing wavelet function, the method needs manual intervention.

\section{Conclusion}

In this study, we proposed a damage location method for tower structures with a cross-correlation function and second generation wavelets. In particular, the introduction of vector angle similarity measure to choose a suitable wavelet improves the accuracy of damage location, such a practice does not appear in other literature too. The method makes it possible to distinguish numerous damage patterns with only a limited number of training samples. The results showed that the damage bars could be recognized accurately in a controlled detecting cost.

Acknowledgement: This work was supported by the Innovative research group project (grant number 51421064) and general project (grant number 51578114) of National Natural Science Foundation of China and general project of Liaoning Education Department. The authors would like to thank these financial supporters.

\section{References}

[1] Stubbs N, Osegueda R. Global Non-Destructive Damage Evaluation in Solids[J], The international journal of analytical and experimental modal analysis, 1990, 5, pp 67-79.

[2] Guoqiang Li, Yuansen Liang, modal curvature in sheet metal structural dynamic detection of vibration[J], Testing and Diagnosis, June 2004, Volume 24, Issue 2.

[3] M. R. Hernandez-Garcia, S. F. Masri, R. Ghanem, E. Figueiredo, and C. R. Farrar, An experimental investigation of change detection in uncertain chain-like systems[J], Journal of Sound and Vibration, 2010, vol. 329, No. 12, pp 23952409.

[4] Suwen Chen, Guoqiang Li. Artificial neural networks in structural damage identification. Vibration[J], Measurement and diagnosis, 2001, Vol.21, No.2:pp116-124.

[5] Long Liu, Guang Meng. Vector regression algorithm applied research in the beam structural damage diagnosis support[J]. Vibration and Shock, 2006, Vol.25, No.3: pp99-100.

[6] R. J. Allemany and D. L. Brown, "A correlation coefficient for modal vector analysis,” in Proceedings of the lst IMAC, pp. 100-116, 1982.

[7] Lei Jiayan, Qianfeng Yao, Lei Ying, et al. Experimental analysis of structural damage identification based on random vibration response of the cross-correlation function[J]. Vibration and Shock, 2011, Vol.30, No.8: 221-224, 236. 
[8] Yu Zhefu, Hou Linsheng, Zhang Lei, Grid Structure Damage Location Base on Cross-correlation Function [C]. The 4nd international conference on civil, architecture and building materials, 2014, pp. 1083-1086.

[9] Sweldens W. schroder P. Building your own wavelets at home [DB/OL]. http://cm.bell-labs.com/who/wim/papers.html/at home. 1998-01-05.

[10] Cohen, A., Daubechies, I., and Feauveau, J. Bi-orthogonal bases of compactly supported wavelets, Comm. Pure Appl. Math. 45, 1992, pp. 485-560.

[11] Daubechies, I.Ten lectures on wavelets. CBMS-NSF Regional Conf. series in Appl. Math., Society for Industrial and Applied mathematics, Philadelphia, PA, 1992.

[12] Daubechies, I. Orthonormal bases of compactly supported wavelets II : variations on a theme. SIAM J. Math. Anal. 24, 2, 1993, pp.499-519.

[13] Deslauriers, G., and Dubuc, S., Interpolation dyadique. In fractals, dimensions non entieres et applications. Masson paris, 1987, pp.44-55.

[14] Donoho, D. L. Interpolating wavelet transforms. Preprint, Department of Statistics, Stanford University, 1992.

[15] Donoho, D. L. Smooth wavelet decompositions with blocky Coefficient Kernels. In[29]. pp.259-308.

[16] Sweldens W. the lifting scheme: A custom-design construction of biorthogonal wavelets [J]. applied and computational harmonic Analysis. 1996. vol.15, no.3, pp.186-200.

[17] Lu Liang, Long Yuan, Xie Quanmin, Li Xinghua, Ji Chong, Zhao Changxiao Decomposition and energy distribution of blasting vibration signal based on second generation wavelet packet. Explosion and shock waves, vol.33, No.2, 2013, pp.140-147.

[18] Liu Chuncheng, Liu Jiao, Li Hongnan, "Damage identification for transmission tower based on support vector machines," Journal of basic science and engineering, vol.18, no.4, pp.616-625, 2010.

[19] He Haoxiang, Yan Weiming, and Zhou Xiyuan, “Application of wavelet support vector machine to structural damage detection," Journal of vibration, measurement \& diagnosis, vol. 27, no. 1, pp. 53-57, 2007.

[20] Yu Zhefu and Huo Linsheng, "A practical method for grid structures damage location.” Journal of Sensors, Special Issue: Smart Sensing Technologies and their Applications in Civil Infrastructures, 2015.

Yu Zhefu received PhD degree from Dalian maritime University, City: Dalian, Country: China, in 2011. Now he works at Dalian maritime University. His current research interests include mode distinguish and fault diagnosis.

Huo Linsheng now works at Dalian University of Technology, City: Dalian, Country: China. His current research interests include structural vibration control and fault diagnosis. 